\title{
Quality assessment of dynamic virtual relighting from RTI data: application to the inspection of engineering surfaces
}

\author{
Abir Zendagui ${ }^{\mathrm{a}}$, Gaëtan Le Goïc ${ }^{\mathrm{a}}$, Hermine Chatoux ${ }^{\mathrm{a}}$, Jean-Baptiste Thomas ${ }^{\mathrm{a}, \mathrm{b}}$, Jochum $\mathrm{P}^{\mathrm{c}}$, \\ Maniglier $\mathrm{S}^{\mathrm{d}}$, Yuly Castro ${ }^{\mathrm{a}}$, Marvin Nurit ${ }^{\mathrm{a}}$, and Alamin Mansouri ${ }^{\mathrm{a}}$ \\ amage et Vision Artificielle Laboratory, ImViA EA 7535, University of Burgundy, Dijon, \\ France \\ ${ }^{\mathrm{b}}$ The Norwegian Colour and Visual Computing Laboratory, Department of computer science, \\ NTNU, Gjøvik, Norway \\ cFrancéclat, Technical department, Besançon, France \\ ${ }^{\mathrm{d}}$ Technical Center for Mechanical Industry (CETIM), Cluses, France
}

\begin{abstract}
This paper aims to evaluate the visual quality of the dynamic relighting of manufactured surfaces from Reflectance Transformation Imaging acquisitions. The first part of the study aimed to define the optimum parameters of acquisition using the RTI system: Exposure time, Gain, Sampling density. The second part is the psychometric experiment using the Design of Experiments approach. The results of this study help us to determine the influence of the parameters associated with the acquisition of Reflectance Transformation Imaging data, the models associated with relighting, and the dynamic perception of the resulting videos.
\end{abstract}

Keywords: Material appearance, Reflectance Transformation Imaging, Psychometric evaluation, Visual assessment

\section{INTRODUCTION}

Controlling the appearance of engineered surfaces is of primary importance for many high added-value industrial products. A major issue is that surfaces are often characterized through geometric specifications, ${ }^{1}$ and the functional relation between these geometric descriptors. ${ }^{2}$ The optical properties of surfaces, and more generally the visual appearance (how they are perceived) are not explicitly considered. ${ }^{3,4}$ Indeed, this interaction and its perception by people is related to a complex multi-physical and multi-scale process. In an industrial context, the Reflectance Transformation Imaging technique can be seen as simplification of BRDF ${ }^{5}$ allowing to estimate the angular reflectance at each point/pixel of the inspected surface. Angular reflectance plays an essential role in the perception of surfaces, ${ }^{6}$ and its capture ${ }^{7-10}$ fills the gap between sensory evaluation and geometric measurement as illustrated in Figure 1. This process is bidirectional: either reproducing a design, i.e. a visual emotion, on the manufactured surfaces and/or, inversely, checking the conformity of sensory visual descriptors during or at the end of the production process.

Reflectance Transformation Imaging (RTI) acquisition consists in acquiring a series of images by a fixed camera positioned orthogonal to the studied surface while varying the light direction. Each image of the series corresponds to an illumination direction. The images are used afterwards in a modelling process to reconstruct the angular reflectance and relight the surface under any arbitrary illumination direction.

The objective of this study is to investigate the relevance of this information for the appearance inspection ${ }^{11}$ of engineered surfaces. A previous study in this direction made it possible to understand the influence of the acquisition and the modeling parameters on the accuracy of the relighting ${ }^{7}$ using the RTI data. This preliminary study highlighted an important limitation in the proposed experiment. This limitation is due to the fact that

S:

Abir Zendagui: E-mail: abir_zendagui@etu.u-bourgogne.fr 


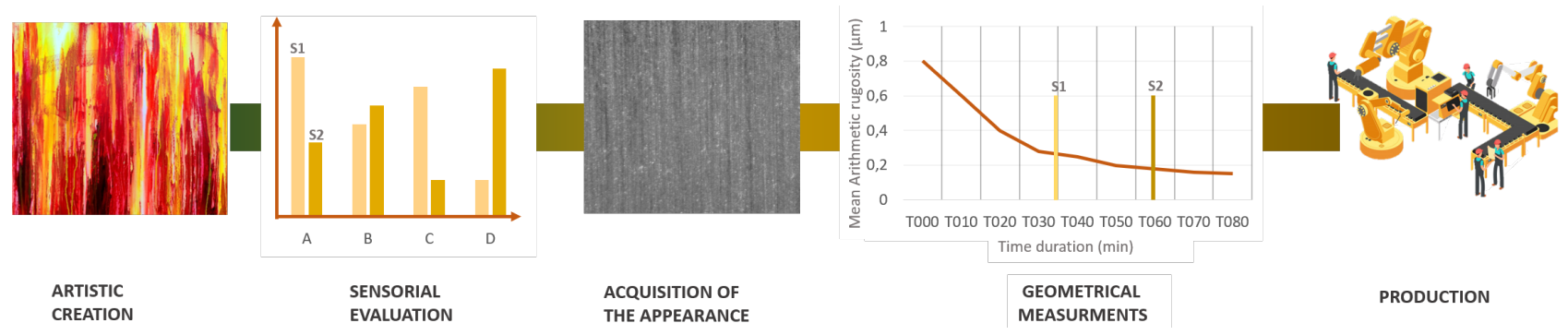

Figure 1. The production line of a high quality manufactured surfaces: from the artistic creation to the production of the surface.

the psychometric evaluation was carried out on fixed images. Each image is associated with a fixed angle and the different study parameters. The assessment is therefore based on static images, which represents a significant and critical difference with the way in which humans carry out this type of evaluation. For example, in the industrial context of a visual sensory assessment, controllers are required to vary the incidence of light by changing the orientation of the surface to increase the robustness of the process. To address this limitation, this study implements the analysis of dynamic relighted surfaces to quantify the impact of the acquisitions parameters closely to the functioning of human perception. To this end, we have implemented a dynamic relighting psychometric experiment, which means the assessment is made from reconstructed videos of the surface appearance in which the light direction varies according to a defined illumination path, consistent with industrial visual control practices, where the controllers are required to follow an exploration protocol, which includes the variation of the azimuth and theta illumination angles $(\theta, \phi)$.

In the present study, the first step aims to determine the acquisition parameters (Exposure time, Gain, Sampling density) per experiment surface (three engineered surface were used with different reflectance behaviour) using the Design of experiment Approach (DoE). The second step aims to evaluate the accuracy of dynamic relighting using RTI data where we noticed a preference of DMD as an approximation model to relight virtually a surface.

\section{METHODS}

The quality of RTI reconstruction depends on several parameters related to both the acquisition and the modeling process. In a non-exhaustive way, significant factors that we have identified are, for example, the acquisition scale, the spatial-angular resolution, the exposure parameters, the illumination wavelength, the implemented angular reflectance model, the number of descriptors implemented in the chosen model, or even the reconstruction light path. The quality of RTI reconstruction also depends on the intrinsic complexity of the surface reflectance behaviour, and interactions are potentially existing and significant between many of these factors. The surface samples, the RTI acquisition device and parameters, and the Design Of Experiment (DOE) proposed method and implementation are presented below.

Experiment Samples. For this study, three engineered surface samples have been selected because they show different reflectance behaviour. These samples are presented in figure 2. They are respectively an industrial paper sample, noted $S_{1}$ in the following. $S_{2}$ is a metallic sand blasted sample (Spectralon $\AA$ SRS-05-020 Diffuse Reflectance Standard), and $S_{3}$ is a glossy metallic sample (brushed-polished surface). $S_{1}$ and $S_{2}$ are diffuse material contrary to $S_{3}$.

RTI acquisition setup and parameters. The acquisitions were carried out with an in-house developed RTI system (figure 3a). The vision system is based on a monochromatic $2 / 3$ active pixel-type CMOS sensor, allowing low-noise acquisitions with a resolution of $12.4 \mathrm{Mp}(4112 \times 3008)$. The camera is equipped with a 

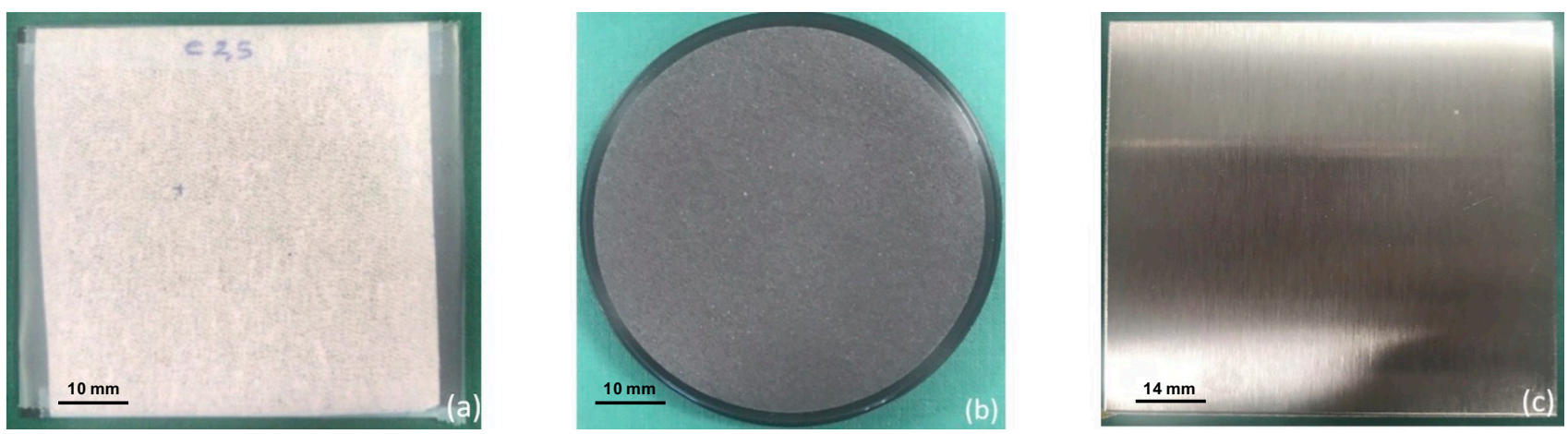

Figure 2. Experiment Samples: (a) $S_{1}$ - Industrial paper (b) $S_{2}$ - Spectralon@ Diffuse Reflectance Standard (SRS-05-020) (c) $S_{3}$ - Metallic brushed/polished surface.

precision micro-imaging modular lens system equipped with a motorized magnification and focus, which makes it possible to adapt the field of view and to quickly focus on the area of interest. Regarding the light source, we use a white uniform light (high power LED) equipped with a lens. In order to carry out RTI acquisitions, the light source is mounted on a motorized hoop allowing to control the angular positioning of the source $(\phi$ and $\theta$ angles). All the RTI acquisition parameters can be controlled with a user interface (figure 3b), which also allows the visualization of acquired data and post-processed features mappings. This software has been developed for the specific needs of this study to allow the automation of RTI acquisition cycles on a surface with different acquisition parameters, such as the zoom factor or the number/density of acquisition positions.

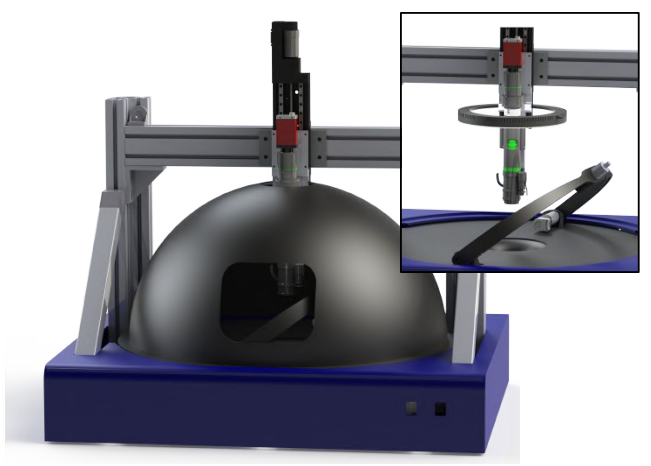

(a) Acquisition device

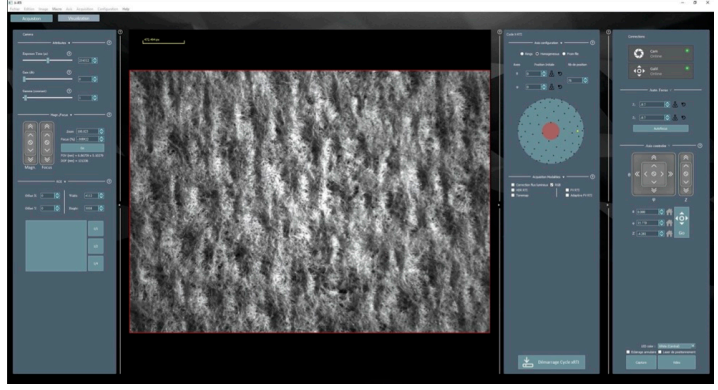

(b) User Interface

Figure 3. Custom RTI acquisition system (ImViA Laboratory), from ${ }^{12}$

A first step (in this study) consisted in defining the optimal setting of the RTI acquisition parameters not retained in the experimental design. Indeed, certain parameters, and particularly those related to illumination, can strongly influence the quality of the data obtained and thus bias the results sought through the psychometric experiment. The evaluation metric chosen to estimate the quality of an RTI acquisition is based on the number of not-measured points in the RTI acquisition, i.e. the sum of the number of under exposed pixels (grey level value of 0) and over-exposed ones (255). After various experiments on the surfaces presented above (figure 2), we chose to study in particular the choice of two parameters for this preliminary step: the angular density of acquisition and the exposure time.

Angular density of acquisition. Indeed, for certain surfaces and particularly anisotropic ones with oriented textures, the acquisition density can influence the chosen metric. For example, for a brushed surface, saturated values are observed when the illumination is orthogonal to the direction of the brushing texture (theta angle), and the number of saturated points will strongly depend on the chosen acquisition density and the width of the solid angle which generates the saturated values when the illumination is close to being orthogonal to the surface texture. The density of acquisition experiment values are 50, 100, 200 and 400, which corresponds to 
[50 - 400] positions homogeneously distributed in the $(\theta, \phi)$ angular space. The results obtained show that an asymptote is reached from a density corresponding to 100 acquisition positions, even for anisotropic surfaces. The tests carried out to determine the optimal adjustment of the acquisition time for each of the surfaces of the experimental plan were therefore carried out with this angular density value.

Exposure time. This parameter has a strong influence on the quality of the data obtained, and its adjustment is generally carried out arbitrarily by the operator in charge of the acquisition. The method then generally consists in choosing an exposure value compromise between the raking illumination angles and higher angles, close to 90 degrees, that induces more over-exposed pixels. For this experiment, we choose 10 exposure values linearly distributed between 2 and 125 milliseconds, which correspond to the upper limit of the lighting time of our device (the led works at high intensity mode, which needs to meet discontinuous operating mode). Moreover, the exposure time value must also be adjusted according to the magnification setting which influences the amount of light received by the sensor. Thus, several images were taken in different level of magnification (from $30 \%$ to $100 \%$ ) to define the optimum exposure time value per magnification. The plot obtained (figure 4) shows the exposure time adjustment values which experimentally makes it possible to obtain a constant luminosity of the images captured at different magnifications with our RTI acquisition setup. As expected, it is observed that the sensitivity of the vision system decreases for large magnifications values. The evolution follows a quadratic law whose least squared approximation is given below in equation 1 (red curve in figure 4). Once the optimal exposure time has been estimated for a surface at an arbitrarily chosen zoom value, this law is then used to determine the optimal exposure time values associated with the different magnification values of the experiment.

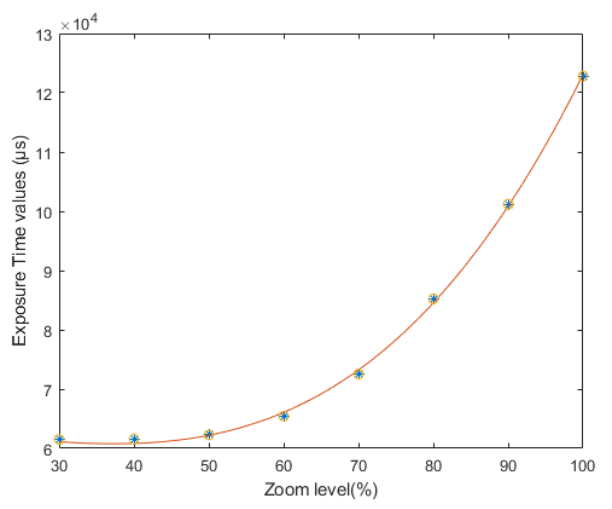

Figure 4. Exposure time $\left(E_{T}\right)$ vs Magnification level $(z)$, estimated from acquisitions on sample $S_{3}$.

$$
E_{T}(z)=0.00006 z^{4}+0.1239 z^{3}-7.1234 z^{2}+64139
$$

The results of this preliminary step are presented in figure 5 for samples $S_{1-3}$. The exposure time used corresponds to the crossing of the curves of the evolution of the percentage of under-saturated points and oversaturated ones, which appears to be a robust value always close to the overall minimum of unmeasured points. The obtained results are synthesized in table 1 and table 2 .

Psychometric experiment. The evaluation is performed through a psychometric experiment by evaluating the quality of reconstruction with the different settings of the Design Of Experiment (DoE) study parameters. Indeed, the quality of RTI reconstruction and its perception depends on several parameters, related to both data acquisition and information modeling and reconstruction. There are, in a non-exhaustive way: the acquisition scale, spatial and angular resolution, the lighting exposure time, the wavelength of light, the reconstruction model, the number of decomposition descriptors, the scale of decomposition, and the reconstruction light path, etc. The quality of RTI reconstruction also depends on the complexity of the surface reflectance behaviour. In this study, we have chosen, based on previous experiments, to focus on 4 parameters that seem to be particularly significant and difficult to control in the case of the RTI process. These parameters, noted $P_{i}$, are: 


\begin{tabular}{c|c|c|c} 
& $S_{1}$ & $S_{2}$ & $S_{3}$ \\
\hline Gain & 0 & 10 & 18 \\
\hline Magnification $(z)$ & 100 & 100 & 100 \\
\hline Optimal Exposure Time $\left(E_{T}\right.$, in ms $)$ & 60.65 & 108.21 & 31.07 \\
\hline Mean percentage of NM pixels (\%) & 0.013 & 0.41 & 11.52
\end{tabular}

Table 1. Optimum Exposure time results for samples $S_{1-3}$, with our RTI acquisition system, at magnification value of $100 \%$.

\begin{tabular}{l|l|l|l|l}
\multicolumn{2}{l}{} & $S_{1}$ & $S_{2}$ & $S_{3}$ \\
\hline \multirow{2}{*}{$\begin{array}{l}\text { Optimal Exposure Time } \\
\left(E_{T}, \text { in } m s\right)\end{array}$} & Magnification (40\%) & 32.69 & 61.68 & 60.83 \\
\cline { 2 - 5 } & Magnification (80\%) & 41.78 & 88.25 & 84.55 \\
\hline
\end{tabular}

Table 2. Optimum Exposure time results for samples $S_{1-3}$, with our RTI acquisition system, for the used magnification levels: $40 \%$ and $80 \%$.
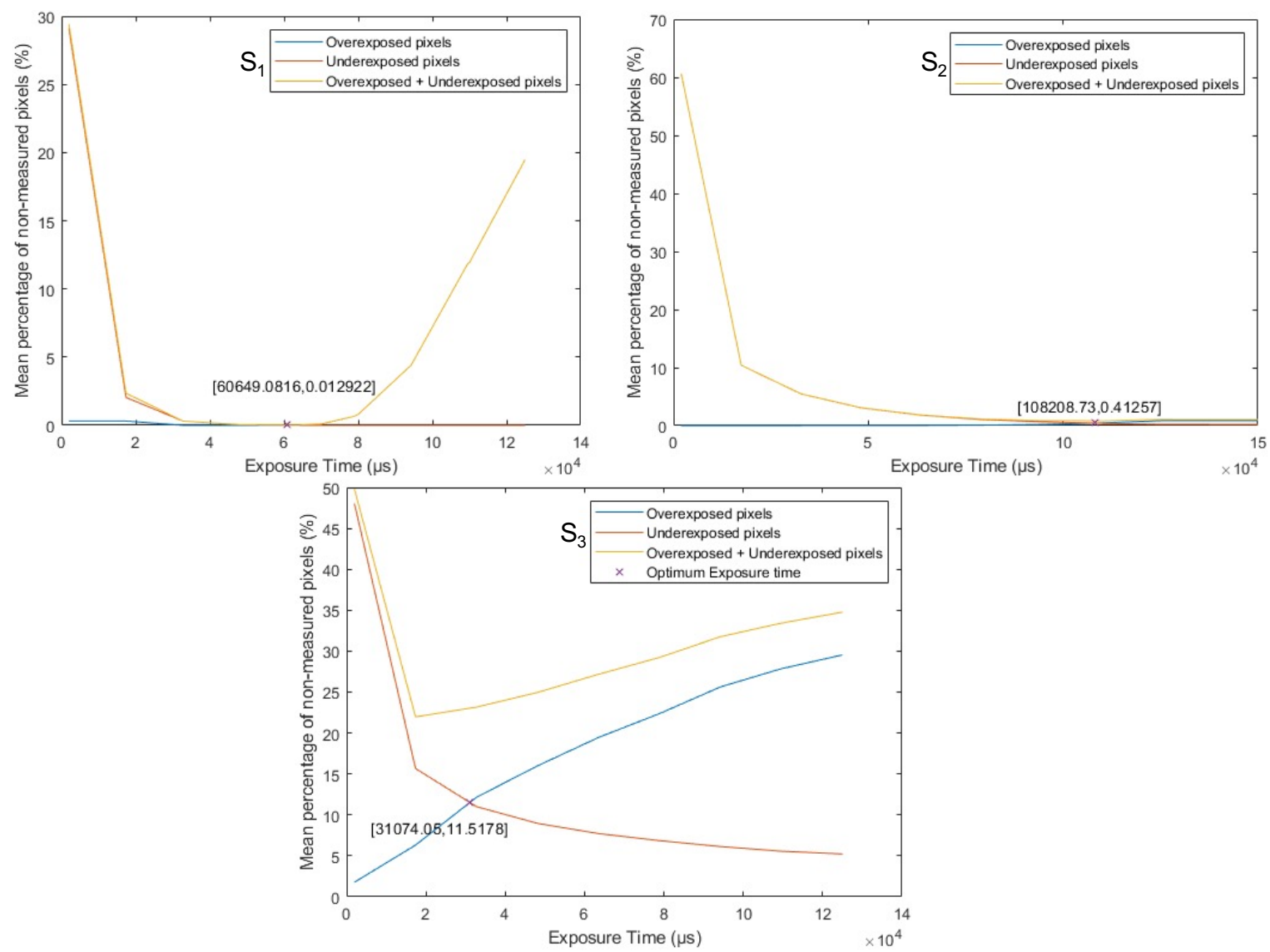

Figure 5. Mean percentage of unmeasured pixels $V s$ Exposure time for RTI acquisitions of Samples $S_{1}, S_{2}$ and $S_{3}(100 \%$ Magnification).

- $P_{1}$, the reconstruction model: three different approximation models of angular reflectance are considered (Polynomial Texture Mappings (PTM) ${ }^{13}$ Hemispherical Harmonics (HSH) ${ }^{14}$ and Discrete Modal Decomposition $(\mathrm{DMD}) .{ }^{15}$

- $P_{2}$, the angular density of acquisition: acquisitions are performed from 50,100, 200 and 400 positions homogeneously distributed in the $(\theta, \phi)$ angular space (see figure 6 ). 
- $P_{3}$, the scale of acquisition: the acquisition system controlling the magnification through a precision motorized lens. Two modalities are defined in the DOE (40\% and 80\%).

- $P_{4}$, the surface reflectance behaviour: three engineered surfaces, already presented above, noted $S_{1-3}$ have been chosen for this experiment.

The influence of the different light path on the reconstruction is not investigated in this study. The light path was defined and kept constant over all experiments, as shown in Figure 6a. The assessment is performed through a psychometric experiment where the participants were asked to choose the most similar video to the reference one. The reference video is associated to a light path in Figure 6.a, varying the azimuth and elevation angles $\left(\theta\right.$ : from $0^{\circ}$ to $359^{\circ}, \phi$ : from $6^{\circ}$ to $\left.30^{\circ}\right)$ and constructed from a dense raw RTI acquisition. The relighted video frame's are obtained from the virtual re-lightening of the surface in each light position of the light path used in the reference video.
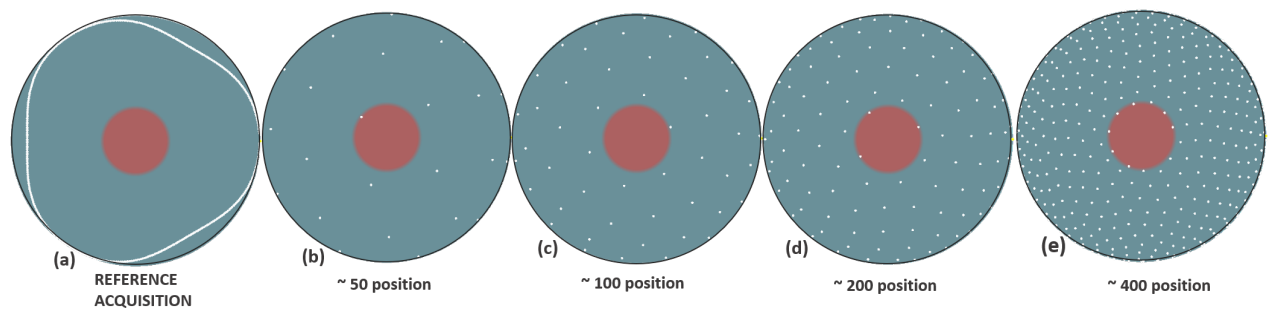

Figure 6. a) Experiment light path: 397 positions (b-d) position of the RTI acquisitions for respectively 50, 100 and 200 positions respectively.

The original and the relighted videos were simultaneously displayed to the experiment screen divided into four parts (see Figure 7). The top-left tagged for the original video through all the experiment. The three other parts are randomly positioned relighted video from parameter $P_{3}$. All the other parameters are fixed per sequence. Each parameter combination from the DoE is projected twice in the experiment. The experiment is divided into two parts: training and main session. The training session sequences ( 5 sequences) were chosen based on their temporal and spatial information, so users span the full range of temporal and spatial information of the test sequences in the experiment conditions. The video sequences duration is $13 \mathrm{~s}$ and randomly projected for each user. The psychometric experiment was designed using MATLABß Design app and started with a data collection of participants information using a text form with: name, last name, age, research field and gender for statistics reasons. The form is followed by a text window to explain instructions and the task. Participants were accompanied in the first step (training session) and allowed to ask questions for a better comprehension of the instructions. The participant's choice is expressed using the keyboard's arrows (up, down, left arrows) and validate their choice using the return button to pass to the next sequence.

\section{RESULTS AND DISCUSSION}

The experiment was held in a dark room, nine volunteers (from the ImViA laboratory team) have participated (7 males, 2 females) with a mean age of 31 years old. All participants have a good visual acuity with correction if needed. All of them have an expertise in image processing and computer vision. The psychometric experiment took an average time of 20.86 minutes per participant, divided to 3.74 minutes for the training session and 17.11 minutes for the main session. The results are presented in the form of heat maps (see figure 8, where the grey cells represents the unmeasured data or outliers), for each of the samples of the study separately, and globally (by averaging the results obtained with all the samples).

The overall results show a significant difference between the appearance reconstruction methods used for RTI relighting. We observe that the PTM reconstruction technique is very rarely chosen (less than $5 \%$ of choices in average) as being the most similar to the reference video. The HSH technique is located in an intermediate way, whereas the Discrete Modal Decomposition is chosen, on average, much more often (approximately $60 \%$ of choices in average) as being the one which offers a perception closest to the reference video. These data confirm 


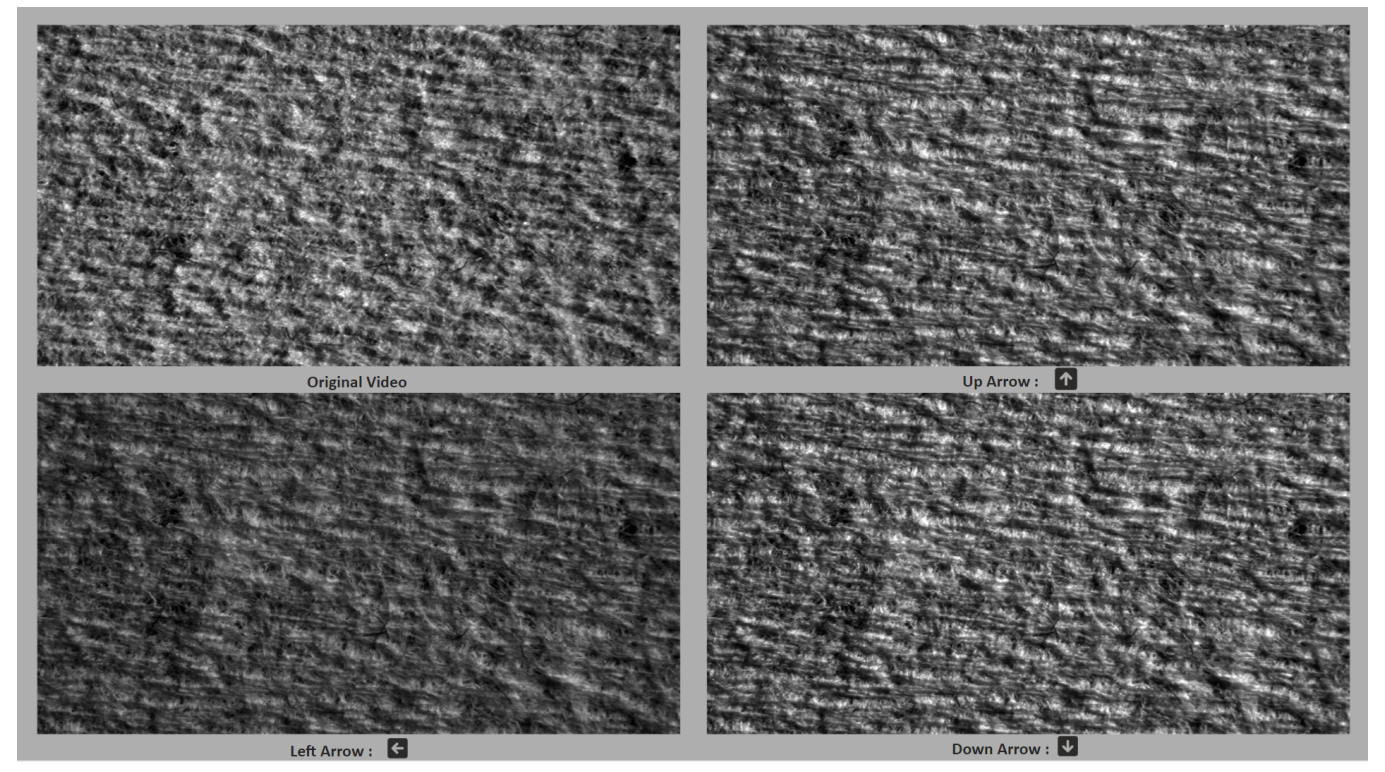

Figure 7. Psychometric experiment: User Interface

the results obtained in the psychometric experiment carried out from static images. ${ }^{7}$

Concerning the effect of the angular density of acquisition, it is observed that the performance of the PTM relighting is globally stable (at a very low level) whatever the angular density of sampling of the RTI acquisition. However, even though outliers were removed from the experiment for the angular density of 50 (the lowest), the results associated with this value seem to show an increase in the performance of the PTM method for this type of acquisition (i.e. with a limited number of acquisition points).

For the HSH method, there is a tendency for the performance of the method to decrease when the acquisition sampling is increased, and vice versa for the DMD method. This can be explained by the lower number of descriptors, i.e. basis vectors, used with the HSH technique (16) than with the DMD technique (50). Indeed, this higher number of descriptors allows, for dense acquisitions, a better approximation of the local variations (higher frequencies) of the angular reflectance in each pixel.

We also observe that the average performance of the HSH technique decreases when the magnification increases, and vice versa for the DMD technique. This tendency is significant in the overall results heat map. For example, for the acquisition density of 100 , the HSH method is chosen on average at $40.74 \%$ with a magnification of $40 \%$, and this percentage is $33.33 \%$ with a magnification of $80 \%$, while the DMD method is chosen. at $55.55 \%$ at $40 \%$ magnification and $62.81 \%$ at $80 \%$ magnification. In our opinion, this is explained by the fact that the sensor integration effect is directly related with the size of the acquired pixel. This integration effect produces a smoothing of the information. Thus, for small acquisition scales, the complexity of the measured angular reflectance tends to increase, which explains the observed increase in performance of the DMD method in comparison with HSH relighting.

Finally, the analysis of the results surface sample by surface surface sample also highlights significant elements in terms of similarity of perception with the reference video. In particular, it is observed that the sample $S_{2}$ leads to a similar evaluation between HSH and DMD relighting techniques, with a slight advantage to HSH. Conversely, the samples $S_{3}$ and $S_{1}$ show a very significant difference between the two techniques, particularly the sample $S_{3}$ where the DMD technique is preferred up to four times more often (for example, for the angular density of 100, the HSH obtains $19.45 \%$ of preferences, while DMD obtains $77.77 \%$ ). These results show that the complexity of angular reflectance can come from both variations in intensity (local specularities, case of sample $S_{3}$ ) and directionality (anisotropic texture, case of samples $S_{1}$ and $S_{3}$ ). In this case, advanced methods, such as DMD, make it possible to improve the perceived quality of RTI reconstructions. Conversely, for surfaces with simpler 
behavior in terms of reflectance, i.e more Lambertian, without directionality (case of the standardized reflectance surface, manufactured by very homogeneous sandblasting), methods such as the HSH technique provide good modeling performance.
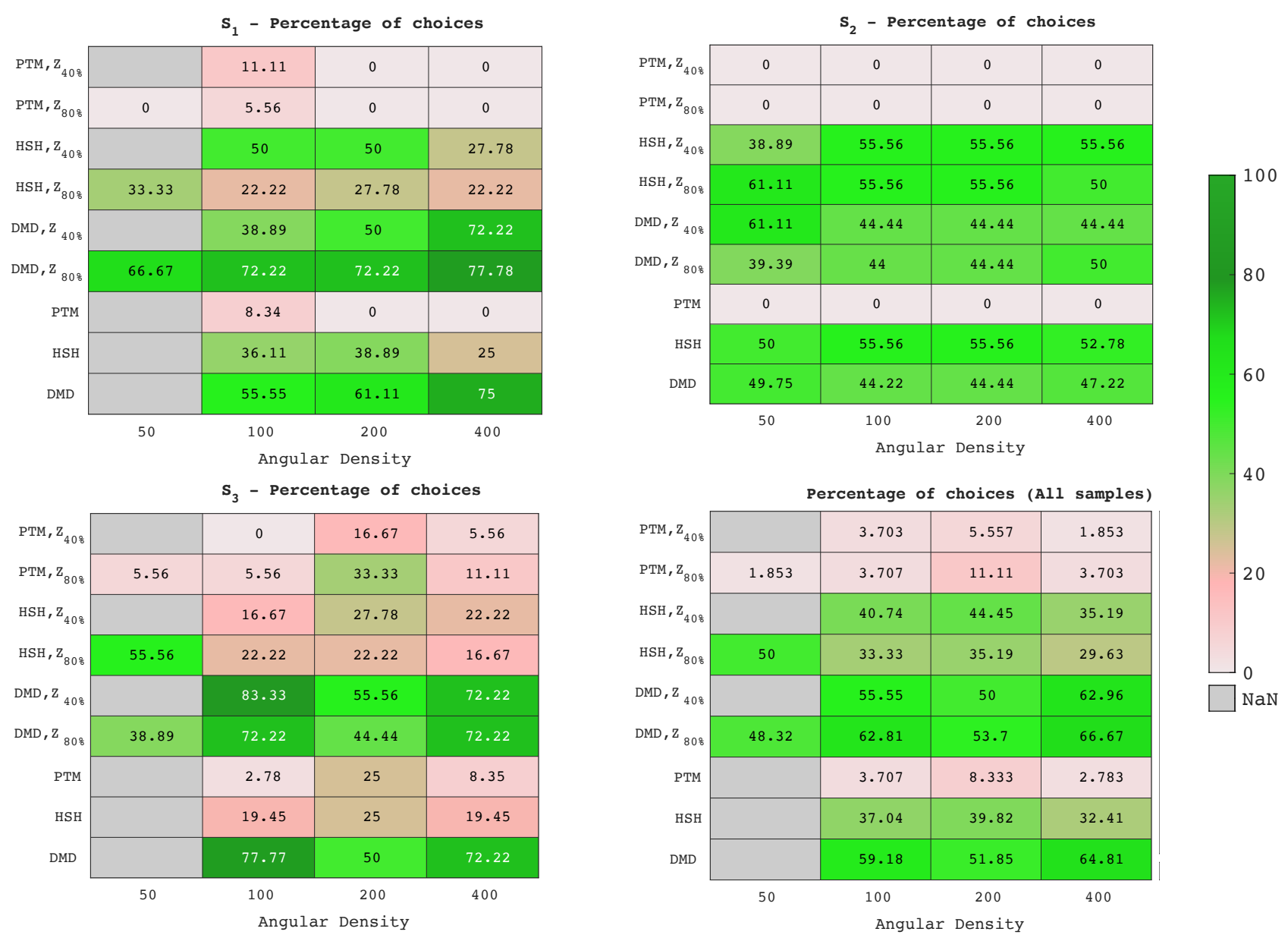

Figure 8. Psychometric experiment: Heat Maps of the percentage of choices for samples $S_{1-3}$

\section{CONCLUSION}

This article firstly presented a method for setting the optimal acquisition parameters in RTI. In a second time it presented a psychometric evaluation of the perceived quality of dynamic appearance reconstruction (relighting) from RTI acquisitions on manufactured surfaces. The studied parameters are the angular density of sampling, the measurement scale and the reconstruction model. Three models are studied (PTM, HSH and DMD). In addition, the psychometric experiment is carried out on three surface samples with distinct reflectance behavior (paper, sandblasted metal, and brushed / polished metal). The results show the importance of the choice of the reconstruction model for the perception of the RTI relighting. The DMD reconstruction model shows the best perceptual performance of the proposed RTI video reconstructions. In addition, a strong interaction is observed between the angular acquisition sampling, the measurement scale, and the choice of the reconstruction model. This study shows that the use of the RTI approach in an industrial context to aid the evaluation of the visual quality of surfaces is relevant and promising, but it is necessary to investigate and control the influence of the parameters of acquisition and modeling in terms of perception. The influence of the light path should also be considered. 


\section{ACKNOWLEDGMENTS}

This work benefited of the funding of French National Research Agency (ANR) through the project NAPS (https://anr.fr/Projet-ANR-17-CE10-0005).

\section{REFERENCES}

[1] Lemesle, J., Robache, F., Le Goic, G., and Mansouri, A., "Surface reflectance: An optical method for multiscale curvature characterization of wear on ceramic-metal composites," Materials 13, 1024 (2020).

[2] Le Goic, G., [Geometric quality and Appearance of surfaces: Local and global approach], Doctoral dissertation, University of Savoie Mont-Blanc (2012).

[3] Pernkopf, F., "3d surface acquisition and reconstruction for inspection of raw steel products," Computers in Industry 56, 876-885 (2005).

[4] Coules, H., Orrock, P., and Seow, C., "Reflectance transformation imaging as a tool for engineering failure analysis," Engineering Failure Analysis 105, 1006 - 1017 (2019).

[5] Obein, G., Audenaert, J., Ged, G., and Leloup, F., [Metrological issues related to BRDF measurements around the specular direction in the particular case of glossy surfaces], Proc. SPIE 9398, Measuring, Modeling, and Reproducing Material Appearance (2015).

[6] Malzbender, T., Gelb, D., Wolters, H., and Zuckerman, B., "Enhancement of shape perception by surface reflectance transformation," HP Labs Technical reports HPL-2000-38R1, 1-3 (2000).

[7] Zendagui, A., Thomas, J. B., Le Goic, G., Castro, Y., Nurit, M., Mansouri, A., and Pedersen, M., "Quality assessment of reconstruction and relighting from rti images: Application to manufactured surfaces," in [2019 15th International Conference on Signal-Image Technology Internet-Based Systems (SITIS)], 746-753 (2019).

[8] Pintus, R., Dulecha, T., Jaspe, A., and Giachetti, A., [Objective and Subjective Evaluation of Virtual Relighting from Reflectance Transformation Imaging Data], Proc. EG GCH, Eurographics Workshop on Graphics and Cultural Heritage (2018).

[9] Giachetti, A., Ciortan, I., Daffara, C., and Pintus, R., [Multispectral RTI Analysis of Heterogeneous Artworks], Proc. EG GCH, Eurographics Workshop on Graphics and Cultural Heritage (2017).

[10] Ponchio, F., Corsini, M., and Scopigno, R., "Relight: a compact and accurate rti representation for the web," Graphical Models 105, 101040 (2019).

[11] Ravimal, D., Kim, H., Koh, D., Hong, J. H., and Lee, S.-K., "Image-based inspection technique of a machined metal surface for an unmanned lapping process," International Journal of Precision Engineering and Manufacturing-Green Technology 7(3), 547-557 (2020).

[12] Nurit, M., Goic, G. L., Lewis, D., Castro, Y., Zendagui, A., Chatoux, H., Favrelière, H., Maniglier, S., Jochum, P., and Mansouri, A., "Hd-rti: an adaptive multi-light approach for the visual quality assessment of manufactured surfaces," Submitted to Computers in industry (2021).

[13] Malzbender, T., Gelb, D., and Wolters, H., "Polynomial texture maps," Proc. SIGGRAPH'01, Computer graphics and interactive techniques, 519-528 (2001).

[14] Gautron, P., Krivanek, J., Pattanaik, S., and Bouatouch, K., "A novel hemispherical basis for accurate and efficient rendering," Proc. ESGR'04 Papers, Eurographics conference on Rendering Techniques , 321-330 (2004).

[15] Pitard, G., Le Goic, G., Mansouri, A., and Favreliere, H., "Reflectance-based surface saliency," Proc. IEEE ICIP, International Conference on Image Processing , 445-449 (2017). 